\title{
A Quick and Painless Reminder: The Pharmacotherapy of Rheumatoid Arthritis in Primary Practice
}

\author{
Linda Brand, a De Wet Wolmarans, ${ }^{\text {a* Sarel J Brand }}{ }^{\mathrm{b}}$ \\ a Center of Excellence for Pharmaceutical Sciences, Division of Pharmacology, Faculty of Health Sciences, North West-University, \\ Potchefstroom, South Africa \\ b Unit for Environmental Sciences and Management, North-West University, Potchefstroom, South Africa \\ *Corresponding author, email: dewet.wolmarans@nwu.ac.za
}

\section{Abstract}

Rheumatoid arthritis, an auto-immune disorder, is characterised by chronic inflammation of the joints, synovial hyperplasia and bone erosion. These pathological features are promoted by a synovial microenvironment featuring B-cell and T-cell infiltrate, synovial fibroblasts and an intricate network of pro-inflammatory cellular messengers - prominent molecular role-players that represent critical targets in the pharmacotherapy of the disease. This review offers a brief overview of the etiopathology of rheumatoid arthritis while focussing on the practical aspects of methotrexate and glucocorticoid use that are of relevance for primary practice.

\section{Fundamentals of Rheumatoid Arthritis}

Rheumatoid arthritis (RA), an auto-immune disorder with unknown aetiology and a chronic and degenerative nature, is associated with synovitis (inflammation of the synovial membranes) and the eventual deterioration of cartilage and erosion of bone due to hyperosteoclastic activity ${ }^{1}$ extending even to extra-articular organs and tissue, resulting in inter alia vasculitis, rheumatoid lung disease, and pericarditis. ${ }^{2}$ A family history of RA has considerable value in predicting the development of the condition in first degree relatives, increasing an individual's odds of suffering from RA up to fourfold $^{3}$ and, combined with genetic (e.g. the HLA DRB1 alleles) and environmental (e.g. cigarette smoking, a diet lacking sufficient omega-3 fatty acids and exposure to infectious agents) risk factors, plays an important role in development and progression of the disease. ${ }^{4}$

Early investigations into the pathophysiology of RA included the discovery of rheumatoid factor (RF) - an antibody in part produced by B-1 lymphocytes - in the blood of patients suffering from RA. ${ }^{5}$ Another prominent characteristic of the autoimmune response in RA patients is the presence of anti-cyclic citrullinated peptide antibodies (ACPAs) ${ }^{6}$ which may precede the onset of disease by up to ten years ${ }^{7}$ - probably indicating that immune activity responsible for triggering the pathology occurs long before eventual symptom development and diagnosis. ${ }^{8}$ Indeed, ACPAs have the most accurate predictive value among antibodies produced in RA. ${ }^{9}$ Considering the presence of these factors, RA is characterised as an auto-immune disorder. However, although the presence of $\mathrm{RF}$ is usually predictive of a more severe disease progression (i.e. more severe joint damage and increased mortality, ${ }^{8,10,11}$ only $75 \%$ of patients (depending on disease stage) are seropositive for the presence of either RF or ACPAs, while up to $80 \%$ of patients test positive for both factors. ${ }^{5,10}$ Regrettably, these factors may also be present in patients suffering from unrelated chronic inflammatory conditions (and even some healthy individuals). Moreover, the possibility of seronegative conversion in patients previously presenting with RF is also highly likely subsequent to successful treatment strategies., , $^{810}$ As such, RA patients may or may not express these antibodies and the presence thereof is not mandatory or even definitive in the diagnosis of RA. They do however remain valuable tools in diagnostic and prognostic considerations. In addition to the presence of auto-antibodies, T-cell and B-cell infiltrate in the synovium also presents a prominent feature in RA. ${ }^{8}$

ACPAs may induce pathogenicity by indirectly activating macrophages (eliciting a pro-inflammatory cytokine response), ${ }^{12}$ and osteoclasts (via tumour necrosis factor (TNF); promoting bone resorption and explaining the increased presence of bone damage in ACPA-positive patients) ${ }^{13}$ and form immune complexes that are able to interact with and potentiate the effects of RF, e.g. cytokine activation and direct macrophage activation. Excessive pro-inflammatory cytokines, e.g. IL-1 and TNF-a, drive the deleterious mechanisms involved in the pathology of RA and it is evident that cells belonging to both the innate (e.g. macrophages) and adaptive (e.g. B and T lymphocytes) immune systems not only interact with one another, but also with resident cells (i.e. synovial fibroblasts). ${ }^{14}$ Subsequently, an array of cytokines are produced that affect the recruitment, retention, and differentiation of infiltrating leukocytes, ${ }^{15}$ producing the inflammatory processes observed in RA. ${ }^{1}$

Aberrant cellular and humoral immune responses therefore forego the presence of autoantibodies, followed by synovial concentration of T- and B-cells. With time, synovial hyperplasia develops due to macrophage invasion, promoting proliferation of synovial fibroblasts. ${ }^{16}$ Continued erosion of cartilage promotes synovial hyperplasia, consequently leading to 
the development of invading pannus tissue ${ }^{17}$ and increased fibroblast and osteoclast activity leads to the destruction of cartilage and surrounding bone. ${ }^{15-17}$ Additionally, synoviocytes produce enzymes, e.g. collagenase and gelatinase, that lead to the detriment of structural matrices. ${ }^{18}$ As a result, RA is thus characterised by a complex interplay of several molecules creating a microenvironment in synovial tissue and/or joints that promotes on-going and debilitating chronic inflammation. Several interactions, e.g. between haematopoietic cells (e.g. lymphocytes) and fibroblasts, T-cells and fibroblasts and T lymphocytes with macrophages and B-cells, contribute to the persistence of inflammation by producing signals that encourage white cell survival and retention. ${ }^{14}$

Considering the above, it is important to note that therapies targeting $\mathrm{B}$ - and T-cell mediated mechanisms provide superior results in patients that are also ACPA- and RF-positive compared to seronegative patients. This, however, is often not the case for TNF-, IL-6- and IL-1-targeted therapies. The adaptive immune response therefore mainly assists in the pathogenesis of seropositive patients while the intricate cytokine-involving processes are an almost universal feature in RA. ${ }^{19}$

\section{Current Pharmacotherapeutic Approaches to Rheumatoid Arthritis}

Briefly, the goal of pharmacotherapy is to reduce the pain, swelling and stiffness of the joints, preserve joint function, slow destructive joint changes, prevent systemic complications and improve the patient's ability to perform his/her daily activities. In this regard, the rapid diagnosis of RA is essential. Indeed, a relatively narrow window of opportunity ( $3-6$ months) from the onset of joint swelling and initiation of a treat-to-target approach with tight monitoring and control exists that may increase the likelihood of remission and lessen damage to the joints. ${ }^{20}$ The three main groups of drugs used for the treatment of RA are the non-steroidal anti-inflammatory drugs (NSAIDs), corticosteroids and disease-modifying antirheumatic drugs (DMARDs). NSAIDs reduce joint pain and swelling, but have no impact on disease progression, while short-term use (<3months) of the corticosteroids can rapidly reduce joint inflammation. However, the only drugs that may actually slow disease progression are the DMARDs. ${ }^{21}$ That said, as the use of biological DMARDs is generally initiated by specialists, the current paper will only briefly summarise key aspects of this drug class while focussing on novel aspects pertaining to the use of glucocorticoids and methotrexate that are of relevance in primary practice.

\subsection{A General Overview of Disease Modifying Anti-Rheumatic Drugs (DMARDS)}

Contrary to earlier guidelines, treatment with the DMARDs is initiated concurrent with diagnosis of RA as they have been demonstrated to reduce the risk of permanent damage to the joints. ${ }^{20,22}$ DMARDs can broadly be divided into the traditional/ synthetic non-biological DMARDs and the recently introduced biological agents, i.e. monoclonal antibodies that still mainly target pro-inflammatory cytokines, e.g. TNF-a; however, antibodies not directed at TNF-a also exist. While the traditional
DMARDs can be administered orally or parenterally, the biological DMARDs can only be injected under the supervision of qualified professionals. The biological DMARDs have a faster onset of action compared to the synthetic DMARDs and as they only target a specific element of the inflammatory response, they do not compromise the entire immune response. However, the high cost and potential risk of severe hypersensitivity reactions limit their use to patients not responding adequately to the traditional DMARDs. ${ }^{23,24}$ In this regard, a trial of at least 6 months on traditional DMARDs without adequate response may be an indication of therapeutic failure. ${ }^{24}$

First-line non-biological DMARDs include methotrexate, hydroxychloroquine, sulfasalazine and leflunomide. Other DMARDs, now seldom used because of their higher toxicity and less than adequate efficacy, include gold (orally as Auranofin \% Ridaura ${ }^{\circ}$ or intramuscularly as Myochrysine ${ }^{\circ}$ ), minocycline, azathioprine (Imuran ${ }^{\circ}$ ) and cyclosporine (Table 1). ${ }^{25}$ The biological agents with disease-modifying activity include the anti-TNF-a group of drugs viz. etanercept (Enbrel $\left.{ }^{\circ}\right)$, infliximab (Revellex ${ }^{\circ}$ ), adalimumab (Humira'), certolizumab and golimumab (Simponi"). Non-TNF-a inhibitors of the immune system include abatacept (Orencia ${ }^{\circ}$ ), that modulates T-cell activation, as well as tocilizumab (Actemra ${ }^{\circ}$ ) and recently FDA-approved sarilumab ${ }^{1}$ that act as IL-6 receptor antagonists. Rituximab, an anti-CD 20 monoclonal antibody that depletes peripheral B lymphocytes, and anakinra, an IL-1 receptor antagonist, can also be used. ${ }^{23}$ Another novel subset of DMARDs (targeted synthetic DMARD) is the orally administered janus kinase (JAK) inhibitors, e.g. tofacitinib ${ }^{1}$ (not yet registered in SA), that inhibit T-cell proliferation. ${ }^{26}$ Nevertheless, the traditional DMARDs, e.g. methotrexate, are still the first-line choice of therapy with or without the concomitant use of glucocorticoids. ${ }^{27,28}$ However, although glucocorticoids have been in use for several decades, recent recommendations still do not provide clear evidence-based guidance with respect to dose, timing and optimal duration of use, tapering strategies and the determination of appropriate long-term benefit vs risk ratios. $^{29}$

\subsubsection{Methotrexate}

Despite the development and availability of a number of biological DMARDs since the 1990s and more recently the targeted synthetic DMARDs, methotrexate (MTX) is still regarded as the gold standard DMARD. It is widely used in monotherapy or in combination with other synthetic or biological agents. ${ }^{30-32}$ However, as in the case of glucocorticoid (GC) therapy, a major clinical challenge associated with MTX use is treatment nonresponse. In fact, $40 \%$ of patients remain refractory to MTX treatment. As such, attempts to provide suitable alternatives currently focus on understanding the mechanisms involved in MTX-resistance and to identify putative biomarkers that may indicate adequate therapeutic response to MTX and other biologic DMARDs before such treatment is initiated..$^{31,33-35}$

Another recent recommendation regarding MTX therapy pertains to its optimal dosage. Over the past few decades the recommended dosage of MTX has been gradually adjusted upwards and it is currently suggested that the mean dose prescribed in RA should range between 20-25 mg/week..$^{25}$ 
However, as the oral absorption of MTX peaks at 15-20 mg/ week, ${ }^{25}$ parenteral MTX is also used more often. ${ }^{36}$

\section{A Fact Box for Primary Practice: When Risk of Prescribing MTX Outweighs the Benefit}

Considering that the biological DMARDs are primarily reserved for use in specialist practice and that no clear guidelines exist that provide detailed algorithms for the initiation, continuation and cessation of MTX therapy in the primary care setting, a useful approach is to be aware of the signs and symptoms that indicate oral overtreatment and that necessitate immediate dose reductions or complete withdrawal from therapy. These are highlighted below: ${ }^{37}$

- Acute renal failure - expect in $2-4 \%$ of patients. Condition is often seemingly asymptomatic and will usually resolve following MTX withdrawal.

- Severe leukopenia - expect in 1-3\% of patients. Consider risk for secondary infection. Condition resolves within 3 weeks following MTX withdrawal.

- Mucocutaneous pathology, i.e. ulceration, photosensitivity, erythema, urticarial and vasculitis. Expect in all patients. Dose reductions and folic acid supplementation are usually adequate to prevent exacerbation.

- Hepatotoxicity, especially in patients with comorbid hepatitis $\mathrm{B}$ or C, diabetes, obesity and alcohol abuse history. Risk can be reduced, but not reversed with folic acid supplementation.

- Pneumonitis - Occurs in $1 \%$ of patients. Involves T-cell mediated hypersensitivity to MTX administration, resulting in pulmonary inflammation associated with a non-productive cough and dyspnoea. Immediate MTX withdrawal is mandatory.

\subsubsection{The Glucocorticoids}

Corticosteroids (administered either systemically or intraarticularly) are not only cost-effective but elicit potent antiinflammatory and immunosuppressive action. As such, they are still used routinely as part of the first-line strategy in the management of RA. ${ }^{37}$ The importance and efficacy of glucocorticoids (GCs) in RA therapy are also reflected in current guidelines for the initial treatment of RA where the concomitant use of GCs with a traditional DMARD is recommended, followed by another synthetic or biologic DMARD with/without a GC if the treatment target of remission is not reached within 3-6 months. ${ }^{27,28}$ Further, irrefutable clinical trial evidence indicated that prednisone ( $7.5 \mathrm{mg}$ daily) may slow the progression of the disease. ${ }^{22,38}$ In an attempt to provide clearer evidencebased guidelines for the use of GCs in RA, European researchers, supported by the GLORIA project, undertook a systematic literature review of clinical practice guidelines (2011-2015). ${ }^{29}$ The results of this review concluded that GCs at low doses and for a short duration of time are an appropriate treatment option of RA. Considering the benefit vs risk ratio of GC therapy in $\mathrm{RA}$, a positive ratio for long-term (3-6 months or more) low dose ( $\leq 5 \mathrm{mg} /$ day prednisone-equivalent) therapy has been demonstrated, while an increased risk of harm in most patients was found at dosages of $>10 \mathrm{mg} /$ day. ${ }^{28}$ Nevertheless, it is true that, at dosages between 5 and $10 \mathrm{mg} /$ day, patient-specific characteristics (protective as well as risk factors) determine an individual's risk of harm, being dose-dependent and patient-specific. Therefore, overall and GC associated risk and protective factors, e.g. lifestyle and diet, should be taken into account when evaluating the actual current and possible future risk. ${ }^{28,39}$

Among the most worrisome risks associated with long-term, high-dose GC use are the development of osteoporosis, diabetes mellitus, weight gain, cardiovascular disease, secondary infection, and myopathy. ${ }^{28}$ As such, in order to minimise adverse effects while preserving or even bolstering GC anti-inflammatory efficacy, two novel approaches are currently under investigation. Though neither is currently advised as an appropriate therapeutic option, this may change soon. First, the development of liposomal GC molecules (prednisolone, methylprednisolone or dexamethasone) involves enfolding the drug molecules in a core of polyethylene glycol (PEG) stabilised liposomes, thereby attempting to enhance tissue-specific drug accumulation in the inflamed joint following intravenous administration. ${ }^{37,40,41}$ Clinical trials investigating the efficacy and safety of these liposomal GC preparations are ongoing. ${ }^{37,42}$ Indeed, a successful phase II clinical trial confirmed the safety and increased efficacy of liposomal prednisolone compared to traditional prednisolone formulations. ${ }^{43}$ Second, the development of highly selective, dissociated, or modulating GC agonists (abbreviated SEGRA, DAGR, and SEGRM, respectively) is currently underway. Such advances are based on the often oversimplified hypothesis that processes of transactivation (i.e. the synthesis of certain regulatory proteins, e.g. enzymes of gluconeogenesis) are responsible for the majority of adverse reactions, while transrepression (i.e. the synthesis of pro-inflammatory cytokines) is instrumental in the anti-inflammatory and immunomodulating effects of GCs..$^{40}$ In this regard, SEGRAs will primarily induce the desired transrepression activity, but reduce the transactivation effects compared to conventional CGs. ${ }^{40,44,45}$ With respect to the DAGRs, fosdagrocorat - a compound with potent anti-inflammatory activity and minimal effects on bone and glucose metabolism - is currently under clinical investigation for the treatment of RA. ${ }^{11,46,47}$ Again, as is also true for MTX treatment, approximately $30 \%$ of RA patients remain resistant to GC therapy.

\section{A Fact Box for Primary Practice: Glucocorticoids and Comorbidity}

As is true for most auto-immune disorders, RA demonstrates a high degree of comorbidity with other conditions marked by aberrant auto-immune responses, especially type 1 diabetes mellitus, inflammatory bowel disease (IBD) and Hashimoto thyroiditis (HT). ${ }^{50}$ As such, a few aspects must be kept in mind in the treatment of RA associated with established or possible comorbidities.

\section{Prescribing glucocorticoids}

- Due to its broad suppression of the immune system, GCs also form part of the first line therapy for IBD and HT. Dosing schedules for these conditions are guided by the same principles as for RA.

- An aspect that is often of great concern when GCs are initiated is established or possible comorbid type 1 diabetes mellitus. Chronic GC administration is known to impair glucose tolerance. Importantly however, high dose (i.e. $60-80 \mathrm{mg}$ prednisone per day), short term (7-10 days) administration of GCs does not alter glucose metabolism to such an extent that coincidental pathology is triggered. ${ }^{51}$ In fact, patients will benefit from such dosing schedules with respect to its anti-inflammatory properties, without presenting with lasting and irreversible metabolic complications. Moreover, as the inflammatory state underlying RA contributes significantly to impair glucose tolerance, the long term benefit of intermittent high dose GC administration most probably outweighs the risk for secondary metabolic complications. ${ }^{51}$

- The same principle as above applies with respect to the benefit vs risk ratio pertaining to all other pathophysiological constructs that may raise concern, e.g. bone-mineral density, hypertension, glaucoma, and cataracts. However, this does not apply to the risk of contracting infectious conditions, and great care needs to be taken in patients with, or who are at high risk for, infectious disease. 


\section{Concluding Statement}

The current paper provides a brief overview of the currently available DMARDs and focuses on practical aspects of MTX and GC use for RA within the context of primary practice. For a complete review of RA, its etiopathology and treatment, the reader is referred to Bester et al. (2016). ${ }^{52}$

Table 1: Summary of the traditional DMARDs, dosages, adverse effects, and monitoring advice (Adapted from [48])

\begin{tabular}{|c|c|c|c|c|c|}
\hline DMARD & Trade name ${ }^{\circ}$ & Dosage & Adverse effects & Comments & Monitoring \\
\hline 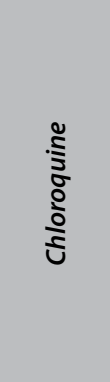 & $\begin{array}{l}\text { Nivaquine } \\
(150 \mathrm{mg}) \\
\text { Plasmoquine } \\
(150 \mathrm{mg})\end{array}$ & $\begin{array}{l}\text { Oral: initially } 150-300 \mathrm{mg} \\
\text { daily, reduced to } 150 \mathrm{mg} \\
\text { daily after } 7-10 \text { days. After } \\
\text { remission is obtained, a } \\
\text { 5-days-a-week regimen } \\
\text { should be attempted. } \\
\text { Recommended dose } \\
2.4 \mathrm{mg} / \mathrm{kg}\end{array}$ & $\begin{array}{l}\text { Macular damage, rash, } \\
\text { diarrhoea }\end{array}$ & $\begin{array}{l}\text { Onset of action may be delayed } \\
\text { for up to } 6 \text { weeks, but should not } \\
\text { be considered as therapeutic } \\
\text { failure until after } 6 \text { months } \\
\text { without response. } \\
\text { Use with caution in renal or } \\
\text { hepatic impairment, alcoholism, } \\
\text { blood disorders, neurological } \\
\text { disorders, psoriasis, pre-existing } \\
\text { visual disturbances }\end{array}$ & $\begin{array}{l}\text { Ophthalmic } \\
\text { examinations before } \\
\text { treatment initiation, } \\
\text { repeat every } 6 \text { months, } \\
\text { regular full blood } \\
\text { counts }\end{array}$ \\
\hline
\end{tabular}

Oral: $2.5 \mathrm{mg} / \mathrm{kg} / \mathrm{day}$ in

2 divided doses, may be

Sandimmun increased gradually after

Neoral 6 weeks to $4 \mathrm{mg} / \mathrm{kg} /$ day.

$(25,100 \mathrm{mg}) \quad$ Discontinue if response

$(25,100 \mathrm{mg}) \quad$ is still inadequate after

IV infusion 3 months. Maintenance:

$(50 \mathrm{mg} / \mathrm{ml}) \quad$ titrate to lowest effective

dose, decreasing by

$0.5 \mathrm{mg} / \mathrm{kg}$ monthly or

bi-monthly
Dose-related

nephrotoxicity,

hepatotoxicity,

neurotoxicity, gingival

hyperplasia and hirsutism
Ciclosporin blood

Potential interaction with drugs levels, kidney and metabolised by cytochrome P450 liver function tests regularly, regular dental examinations
Alanine

aminotransferase (ALT) should be monitored monthly initially and periodically thereafter. Complete blood cell count before therapy starts, 2 weekly for the first 6 months, then 2 monthly. Risk of tuberculosis reactivation
Active RA $10-20 \mathrm{mg}$ once daily. A loading dose of $100 \mathrm{mg} /$ day for the first 3 days may result in a therapeutic response within the first month
Hepatitis, gastrointestinal distress, alopecia, pressure, headaches, dizziness, weight loss, skin rash
(10, $20 \mathrm{mg})$ Rheumalef $(10,20 \mathrm{mg})$
Contraindicated in liver disease

Relative rapid onset of action (2-3 weeks)

Lack of efficacy at $25 \mathrm{mg} /$ week

Methotrexate- Oral: $7.5 \mathrm{mg}$ weekly (as a Lederle single dose or $2.5 \mathrm{mg}$ (2.5 mg) 12 hourly for 3 doses, Abitrexate P\&U Methotrexate CSV $\max 25 \mathrm{mg} /$ week IV or IM: $10 \mathrm{mg}$ once weekly, increased as necessary up to (25 mg/ml)
Myelosuppression, hepatic fibrosis, cirrhosis, pulmonary infiltrates or fibrosis, hematologic, gastrointestinal irritation, stomatitis, rash for 3 months is considered failure of therapy.

May be given with folic acid 1-5 mg/day to reduce adverse reactions, without loss of efficacy.

May precipitate acute attack of gout. Patients with hyperuricaemia should maintain adequate fluid intake, alkalinisation of urine may be useful.

\begin{tabular}{|c|c|c|c|c|c|}
\hline 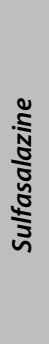 & $\begin{array}{l}\text { Salazopyrin } \\
\text { (500 mg) }\end{array}$ & $\begin{array}{l}500 \mathrm{mg} \text { daily, orally after } \\
\text { food, maximum } 40 \mathrm{mg} / \mathrm{kg} \\
\text { usual maintenance dose } \\
1 \mathrm{~g} \text { twice daily }\end{array}$ & $\begin{array}{l}\text { Myelosuppression, } \\
\text { rash, gastrointestinal } \\
\text { disturbances (take with } \\
\text { food, divide the dose } \\
\text { more evenly throughout } \\
\text { the day). }\end{array}$ & $\begin{array}{l}\text { Antirheumatic effects should be } \\
\text { seen in } 2 \text { months. } \\
\text { Not recommended in renal or } \\
\text { hepatic impairment } \\
\text { Megaloblastic anaemia may } \\
\text { occur, folic acid supplementation } \\
\text { should be prescribed. } \\
\text { Enteric coated tablets may be } \\
\text { useful for long term }\end{array}$ & $\begin{array}{l}\text { Full blood counts, urea } \\
\text { and electrolytes, liver } \\
\text { function tests before } \\
\text { therapy starts and } \\
\text { regularly thereafter }\end{array}$ \\
\hline
\end{tabular}

Full blood counts, urea and electrolytes, liver function tests before therapy starts and regularly thereafter 


\section{References}

1. Angelotti F, et al. One year in review 2017: pathogenesis of rheumatoid arthritis. Clin Exp Rheumatol. 2017;35(3):368-78.

2. Turesson C. Extra-articular rheumatoid arthritis. Curr Opin Rheumatol. 2013;25(3):360-6.

3. Frisell T, Saevarsdottir S, Askling J. Family history of rheumatoid arthritis: an old concept with new developments. Nat Rev Rheumatol. 2016;12(6):335-43.

4. Silman AJ, Pearson JE. Epidemiology and genetics of rheumatoid arthritis. Arthritis Res. 2002;4(Suppl 3):S265-72.

5. Nell V, et al. Autoantibody profiling as early diagnostic and prognostic tool for rheumatoid arthritis. Ann Rheum Dis. 2005;64(12):1731-6.

6. Schellekens $G A$, et al. Citrulline is an essential constituent of antigenic determinants recognized by rheumatoid arthritis-specific autoantibodies. J Clin Invest. 1998;101(1):273-81.

7. Nielen MM, et al. Specific autoantibodies precede the symptoms of rheumatoid arthritis: a study of serial measurements in blood donors. Arthritis Rheum. 2004;50(2):380-6.

8. Scherer HU, Burmester G-R. Adaptive immunity in rheumatic diseases - Bystander or pathogenic player? Best Pract Res Clin Rheumatol. 2011;25(6):785-800.

9. Rantapaa-Dahlqvist $S$, et al. Antibodies against cyclic citrullinated peptide and IgA rheumatoid factor predict the development of rheumatoid arthritis. Arthritis Rheum. 2003;48(10):2741-9.

10. Aletaha D, Alasti F, Smolen JS. Rheumatoid factor, not antibodies against citrullinated proteins, is associated with baseline disease activity in rheumatoid arthritis clinical trials. Arthritis Res Ther. 2015;17(1).

11. Gonzalez A, et al. Mortality trends in rheumatoid arthritis: The role of rheumatoid factor. J Rheumatol. 2008;35(6):1009-14.

12. Clavel $C$, et al. Among human macrophages polarised to different phenotypes, the M-CSF-oriented cells present the highest pro-inflammatory response to the rheumatoid arthritis-specific immune complexes containing ACPA. Ann Rheum Dis. 2016;75(12):2184-91.

13. Harre $U$, et al. Induction of osteoclastogenesis and bone loss by human autoantibodies against citrullinated vimentin. J Clin Invest. 2012;122(5):1791-1802.

14. Buckley CD, et al. Stromal Cells in Chronic Inflammation and Tertiary Lymphoid Organ Formation. Ann Rev Immunol. 2015;33(1):715-45.

15. Buckley $C D$. Why do leucocytes accumulate within chronically inflamed joints? Rheumatology. 2003;42(12):1433-44.

16. Burmester $G$, et al. Rheumatoid Arthritis: pathogenesis and clinical features. EULAR textbook on Rheumatic Diseases. London: BMJ group; 2012:206-31.

17. Redlich K, Smolen JS. Inflammatory bone loss: pathogenesis and therapeutic intervention. Nat Rev Drug Discov. 2012;11:234.

18. Smolian $\mathrm{H}$, et al. Secretion of gelatinases and activation of gelatinase A (MMP-2) by human rheumatoid synovial fibroblasts. Biol Chem. 2001;382(10):1491-9.

19. Stoffer MA, et al. Evidence for treating rheumatoid arthritis to target: results of a systematic literature search update. Ann Rheum Dis. 2016;75(1):16-22.

20. Burmester GR, Pope JE. Novel treatment strategies in rheumatoid arthritis. Lancet. 2017;389(10086):2338-48.

21. Stark SWRNAD. Rheumatoid arthritis. Salem Press; 2003.

22. Weisman MH. Rheumatoid Arthritis. Cary, UNITED STATES: Oxford University Press; 2014.

23. Harmse $L$, Reuter $H$. An overview of the biological disease modifying drugs available for arthritic conditions in South Africa. S Afr Fam Pract. 2016;58(6):6-10.

24. Tarr G, Hodkinson B, Reuter H. Superheroes in autoimmune warfare: Biologic therapies in current South African practice. SAMJ. 2014;104(11):787-91.

25. Jones G, Nash P, and Hall S. Advances in rheumatoid arthritis. Med J Aust. 2017;206(5):221-4.

26. Chatzidionysiou K, et al. Efficacy of glucocorticoids, conventional and targeted synthetic disease-modifying antirheumatic drugs: a systematic literature review informing the 2016 update of the EULAR recommendations for the management of rheumatoid arthritis. Ann Rheum Dis. 2017;76(6):1102-7.

27. Smolen JS, et al. EULAR recommendations for the management of rheumatoid arthritis with synthetic and biological disease-modifying antirheumatic drugs: 2013 update. Ann Rheum Dis. 2014;73(3):492-509.

28. Cindy $S$, et al. Defining conditions where long-term glucocorticoid treatment has an acceptably low level of harm to facilitate implementation of existing recommendations: viewpoints from an EULAR task force. Ann Rheum Dis. 2016(6):952.

29. Palmowski Y, et al. "Official View" on Glucocorticoids in Rheumatoid Arthritis: A Systematic Review of International Guidelines and Consensus Statements. Arthritis Care Res. 2017;69(8):1134-41.

30. Romao VC, Canhao H, Fonseca JE. Old drugs, old problems: where do we stand in prediction of rheumatoid arthritis responsiveness to methotrexate and other synthetic DMARDs? BMC Med. 2013(1).

31. Peres RS, et al. TGF- $\beta$ signalling defect is linked to low CD39 expression on regulatory $\mathrm{T}$ cells and methotrexate resistance in rheumatoid arthritis. J Autoimmun.

32. Smolen JS, et al. EULAR recommendations for the management of rheumatoid arthritis with synthetic and biological disease-modifying antirheumatic drugs: 2016 update. Ann Rheum Dis. 2017;76(6):960-77.

33. Bansard C, et al. Can rheumatoid arthritis responsiveness to methotrexate and biologics be predicted? Rheumatology. 2009;48(9):1021-8.

34. Carini $\mathrm{C}$, et al. Chromosome conformation signatures define predictive markers of inadequate response to methotrexate in early rheumatoid arthritis. J Trans Med. 2018;16(1):18.

35. Hider SL, et al. Can clinical factors at presentation be used to predict outcome of treatment with methotrexate in patients with early inflammatory polyarthritis? Ann Rheum Dis. 2009;68(1):57-62.

36. Gaujoux-Viala CA, et al. Optimal methotrexate dose is associated with better clinical outcomes than non-optimal dose in daily practice: results from the ESPOIR early arthritis cohort. BMJ Publishing Group Ltd; 2017:2054.

37. Gaies $\mathrm{E}$, et al. Methotrexate side effects: review article. J Drug Metab Toxicol. 2012;3(4):1-5

38. Buttgereit F, Bijlsma JWJ, Strehl C. Will we ever have better glucocorticoids? Clin Immunol. 2017.

39. Kirwan JR, T Arthritis, RCL-DGS Group. The Effect of Glucocorticoids on Joint Destruction in Rheumatoid Arthritis. N Engl J Med. 1995;333(3):142-7.

40. Hoes J, et al. EULAR evidence-based recommendations on the management of systemic glucocorticoid therapy in rheumatic diseases. Ann Rheum Dis. 2007;66(12):1560-7.

41. Buttgereit F, Burmester GR, Lipworth BJ. Optimised glucocorticoid therapy: The sharpening of an old spear. Lancet. 2005;365(9461):801-3.

42. Vandewalle J, et al. Therapeutic Mechanisms of Glucocorticoids. Trends Endocrinol Metab. 2017.

43. Barrera $P$, et al. Long-circulating liposomal prednisolone versus pulse intramuscular methylprednisolone in patients with active rheumatoid arthritis in Arthritis and Rheumatism. Hoboken, NJ: Wiley-Liss Div John Wiley \& Sons inc; 2008.

44. Prasad LK, O'Mary H, Cui Z. Nanomedicine delivers promising treatments for rheumatoid arthritis. Nanomedicine. 2015;10(13):2063-74.

45. Schacke $\mathrm{H}$, et al. Dissociation of transactivation from transrepression by a selective glucocorticoid receptor agonist leads to separation of therapeutic effects from side effects. Proc Natl Acad Sci U S A. 2004(1):227.

46. Schäcke $H$, et al. Insight into the molecular mechanisms of glucocorticoid receptor action promotes identification of novel ligands with an improved therapeutic index. Exp dermatol. 2006;15(8):565-73.

47. Huh J, et al. Treatment of generalized anxiety disorder: A comprehensive review of the literature for psychopharmacologic alternatives to newer antidepressants and benzodiazepines. Prim Care Companion J Clin Psychiatry. 2011;13(2).

48. Stock T, et al. Improved disease activity with fosdagrocorat (PF-04171327), a partial agonist of the glucocorticoid receptor, in patients with rheumatoid arthritis: a Phase 2 randomized study. Int J Rheum Dis. 2017;20(8):960-70.

49. Rossiter D. (Ed). The South African Medicines Formulary. Cape Town: Health and Medical Publishing Group; 2016:650.

50. Cooper GS, Bynum MLK, Somers EC. Recent insights in the epidemiology of autoimmune diseases: Improved prevalence estimates and understanding of clustering of diseases. J Autoimmun. 2009;33(3-4):197-207.

51. den Uyl D, et al. Metabolic effects of high-dose prednisolone treatment in early rheumatoid arthritis: Balance between diabetogenic effects and inflammation reduction. Arthritis Rheumatol. 2012;64(3):639-46.

52. Bester FCJ, Bosch FJ, van Rensburg BJJ. The specialist physician's approach to rheumatoid arthritis in South Africa. Korean J Intern Med. 2016;31(2):219-36. 